\title{
Implementation of guidelines for implantable cardioverter-defibrillator therapy in clinical practice: Which patients do benefit?
}

\author{
S. C. Wijers • B. Y. M. van der Kolk • A. E. Tuinenburg • \\ P. A. F. Doevendans • M. A. Vos • M. Meine
}

Published online: 10 April 2013

(C) The Author(s) 2013. This article is published with open access at Springerlink.com

\begin{abstract}
Purpose Based on multiple large clinical trials conducted over the last decades guidelines for implantable cardioverterdefibrillator (ICD) implantations have been evolving. The increase in primary prophylactic ICD implantations challenges us to be critical towards the indications in certain patient populations.

Methods We retrospectively collected patient characteristics and rates of appropriate and inappropriate ICD therapy, appropriate and inappropriate ICD shock and mortality of all patients who received an ICD in the University Medical Center Utrecht (UMCU) over the years 2006-2011.

Results A total of 1075 patients were included in this analysis (74\% male, mean age $61 \pm 13$ years, left ventricular ejection fraction $30 \pm 13 \%$ ); $61 \%$ had a primary indication
\end{abstract}

The questions can be answered after the article has been published in print. You have to $\log$ in to: www.cvoi.nl.

S. C. Wijers · B. Y. M. van der Kolk · M. A. Vos

Department of Medical Physiology, Division of Heart and Lungs,

University Medical Center Utrecht, Utrecht, the Netherlands

S. C. Wijers · B. Y. M. van der Kolk · A. E. Tuinenburg •

P. A. F. Doevendans $\cdot$ M. Meine

Department of Cardiology, Division of Heart and Lungs,

University Medical Center Utrecht, Utrecht, the Netherlands

S. C. Wijers $(\bowtie) \cdot$ B. Y. M. van der Kolk $\cdot$ M. A. Vos

Department of Medical Physiology,

University Medical Center Utrecht, Yalelaan 50,

3584 CM Utrecht, the Netherlands

e-mail: s.c.wijers@umcutrecht.nl

A. E. Tuinenburg • P. A. F. Doevendans • M. Meine

Department of Cardiology, University Medical

Center Utrecht, Heidelberglaan 100,

3584 CX Utrecht, the Netherlands and $58 \%$ had ischaemic heart disease. During a mean follow-up period of $31 \pm 17$ months, 227 of the patients (21\%) received appropriate ICD therapy (149 (14\%) patients received an appropriate ICD shock). Females, patients with a primary prophylactic indication and patients with nonischaemic heart disease experienced significantly less ICD therapy. Only a few patients $(54,5 \%)$ received inappropriate ICD therapy; 33 (3\%) patients received an inappropriate ICD shock. Fifty-five patients died within one year after ICD implantation and were therefore, in retrospect, not eligible for ICD implantation.

Conclusion Our study confirms the benefit of ICD implantation in clinical practice. Nevertheless, certain patients experience less benefit than others. A more patient-tailored risk stratification based on electrophysiological parameters would be lucrative to improve clinical benefit and cost-effectiveness.

Keywords Implantable cardioverter-defibrillator · Primary prevention - Secondary prevention - Ventricular arrhythmia . Mortality

\section{Introduction}

For almost 30 years, implantable cardioverter defibrillators (ICD) have been used in the prevention of sudden cardiac death caused by life-threatening cardiac arrhythmias. In 1984, the first ICD implantation in the Netherlands was performed at the University Medical Center Utrecht (UMCU). Over the last decades, multiple studies, including series of randomised controlled trials, have demonstrated a beneficial effect of an ICD in the prevention of sudden cardiac death [1-8]. In particular, in patients with ischaemic heart disease and impaired left ventricular function, primary 
prophylactic ICD implantation was proven to be effective $[3,6,8]$. In patients with non-ischaemic cardiomyopathy, however, trials had difficulties reaching statistical significance, because of the low incidence of life-threatening tachyarrhythmias in these patients [6]. Guidelines have been evolving over the years incorporating the evidence of recent trials [9-11]. In the last decade the evidence about primary prophylactic ICD therapy produced a steep increase in the number of ICD implantations in Western Europe countries. A shift in indication from predominantly secondary to primary prophylaxis occurred due to a significantly higher number of suitable candidates, which increased further with the broadening of indications in the guidelines of 2008 [9]. However, recent reports express concerns about cost-effectiveness and the benefit-complication ratio [12-15]. The question is in which patients and in how many of them we actually prevent a sudden cardiac death. Critical articles about the sense and non-sense of ICD implantation for primary prophylaxis in certain patient populations have given rise to some doubts making it more difficult to implement and interpret guidelines accurately $[16,17]$. This results in a higher number of nonevidence-based ICD implantations on one hand and a substantial number of patients eligible for ICD implantation, which are not recognised on the other.

We retrospectively collected several patient characteristics for all patients who received an ICD implant in the University Medical Center Utrecht (UMCU) over the years 2006-2011. In this study we give an overview of ICD implantations in this centre and present rates of, and risk factors for, appropriate and inappropriate ICD therapy, appropriate and inappropriate ICD shock and mortality. Subsequently, we evaluate the adherence to international guidelines in clinical practice and provide directions for future indications for ICD implantation.

\section{Materials and methods}

\section{Patient population and parameters}

In our tertiary centre, we retrospectively identified all new ICD implantations from 2006 until the end of 2011. In the determination of different indications, the class I or II recommendations of the 2006 and 2008 ACC/AHA/HRS guidelines were used $[9,18]$. Obtained variables included patient demographics, indication for implantation, New York Heart Association (NYHA) functional class, left ventricular ejection fraction (LVEF), renal clearance, history of diabetes, documented rhythm disorders, QRS duration, medication and device settings.

The LVEF was rated by transthoracic echography, nuclear myocardial perfusion scan, or magnetic resonance imaging (MRI). If more than one modality was available, preference was given to LVEF provided by MRI. To determine renal function at baseline, serum creatinine was used to calculate the glomerular filtration rate (GFR) using the Modification of Diet in Renal Disease (MDRD) formula [19]. History of diabetes and use of medication was also rated at baseline or at least as close as possible to the date of implantation, with a maximum of one month. To allocate the different antiarrhythmic drugs into classes, the Vaughan Williams classification was used [20]. Finally, specific information regarding device programming was retrieved from the last visit to the outpatient clinic.

Data were collected from electronic medical records and implantation reports by the first two authors. Collection of follow-up data for all patients was completed by the end of May 2012.

Implant technique and ICD programming

All devices were implanted by one of our cardiologists in the Cardiac Catheterisation Laboratory of the UMCU. No thoracotomies were performed for implantation and all patients received endocardial leads and subpectoral or subcutaneous device placement. Devices from Boston Scientific (Natick, MA, USA), Medtronic (Minneapolis, MN, USA) and St. Jude Medical (St Paul, MN, USA) were used. Programming of ventricular tachycardia (VT)/ventricular fibrillation (VF) zones, monitor zones and additional programming of antitachycardia pacing (ATP) therapy was decided by patient's cardiologist.

\section{Definitions}

Ischaemic cardiomyopathy as underlying heart condition was defined as either the presence of coronary artery disease, myocardial infarction or both. A history of atrial tachyarrhythmia was documented when patients suffered from atrial fibrillation or atrial flutter, or had experienced episodes of these arrhythmias in the past. We defined ICD therapy as delivery of either antitachycardia pacing (ATP) and/or ICD shock (cardioversion or defibrillation), which was considered appropriate when given in the presence of a ventricular tachyarrhythmia. Therapy given in the absence of a ventricular tachyarrhythmia, but triggered as a result of i.e. supraventricular tachycardia or due to technical disturbances, was defined as inappropriate ICD therapy. For the incidences of either inappropriate or appropriate ICD shock all patients who received an ICD shock were counted regardless of delivery of previous ATP. For incidences of both ICD therapy and ICD shock, each patient is counted once, regardless of the number of events in one patient.

Statistical analysis

Categorical variables were presented as absolute numbers and percentages and continuous variables were expressed as 
a mean with an upper and lower standard deviation. To compare differences between baseline characteristics, the independent sample t-test was used for continuous variables and the chi-square test for categorical variables.

Multivariate Cox regression analysis was used to determine independent risk factors for appropriate and inappropriate ICD therapy, appropriate and inappropriate ICD shock and mortality. Kaplan-Meier curves were constructed to determine cumulative incidence of appropriate ICD shocks. To perform these analyses, SPSS 20.0 (IBM, USA) was used.

\section{Results}

From January 2006 to December 2011, 1075 de novo ICD implantations were performed at the UMCU.

\section{Baseline characteristics}

An overview of the baseline characteristics of the 1075 patients is presented in Table 1. In 2006, 134 ICD implantations were performed; in 2011 this amount had almost doubled $(229,+171 \%)$. ICDs were implanted for primary prevention in 654 patients $(61 \%) ; 626$ (58\%) patients had ischaemic heart disease. There was a $3 / 1$ male versus female ratio. Of all de novo implants almost $30 \%$ consisted of cardiac resynchronisation therapy defibrillator (CRT-D) implantations. The mean age of the total population was $61 \pm 13$ years, the mean LVEF $30 \pm$ $13 \%$, and $78 \%$ used at least one antiarrhythmic agent, particularly beta-blockers (71\%). A history of atrial arrhythmia was seen in $28 \%$ of the patients. During a mean follow-up period of 31 months ( \pm 17 months), 155 patients died.

Ischaemic versus non-ischaemic cardiomyopathy

In Table 1, a distinction was made between patients with ischaemic cardiomyopathy (ICM) and non-ischaemic cardiomyopathy (NICM). The mean age at implant in the patients with NICM was lower ( $55 \pm 15$ versus $66 \pm 10, p<$ $0.001)$ and the distribution between men and women was more equal (male/female ratio of 5.3/1 versus $1.5 / 1$ ). In patients with ischaemic aetiology, the mean LVEF and the GFR were significantly lower and diabetes mellitus type II more prevalent.

Primary versus secondary indication for ICD implantation

The increase in the number of ICD implantations was mainly based on the increase in primary prophylactic implants. The percentage of patients with ICM was significantly lower in the patients with an ICD implanted for primary prevention compared with secondary prevention (54\% and $64 \%$, respectively, $p<0.001)$. LVEF was significantly higher in patients implanted for secondary prevention than in patients with a primary indication, $36 \pm 15 \%$ versus $27 \pm 11 \%$, respectively $(p<0.001)$.

\section{ICD settings}

A single zone (VF only) was installed in $7 \%$ of the patients, two zones (VT+VF) in $80 \%$ and three zones (VT-1, VT-2 and VF) in $10 \%$. In the VT-1, VT-2 and VF zone the mean rates installed were $180 \pm 13,188 \pm 12$, and $226 \pm 17$ beats/min, respectively. In the remaining $3 \%$, data of the ICD settings were not available. In approximately $90 \%$ ATP was programmed (either as single therapy or prior to shock therapy). VT/supraventricular tachycardia (SVT) discrimination algorithms were programmed in the VT zones in all patients. No major differences between monitor and therapy zones were observed between the different subpopulations of patients that experienced ICD therapy.

\section{ICD therapy}

In the total population of 1075 patients, 227 patients $(21 \%)$ received at least one episode of appropriate ICD therapy (149 appropriate ICD shocks (14 \%)) during the mean follow-up period of $31 \pm 17$ months. Inappropriate ICD therapy was seen in 54 patients $(5 \%), 33$ patients $(3 \%)$ received an inappropriate ICD shock.

Cumulative incidence of appropriate ICD shock was $7 \%$ at one year, $16 \%$ at three years and $23 \%$ at five years. When corrected for several baseline characteristics by multivariate Cox regression analysis, a secondary indication, ICM, decreased LVEF $(\leq 25 \%)$ and the male gender were independent predictors for appropriate ICD shock, with hazard ratios (HR) of 1.8 (95 \% CI: 1.3-2.5; $p=0.001), 1.6$ (95 \% CI: 1.1-2.3; $p=0.023), 1.6$ (95 \% CI: $1.2-2.3, p=0.004)$ and 2.3 (95\% CI: $1.4-3.8 ; p=0.001)$, respectively (Fig. 1). Gender showed to be the most important risk factor for appropriate ICD shocks, with men having a more than doubled risk for appropriate ICD shock. When we only analysed patients who had an ICD implanted for primary prophylaxis, the incidences of appropriate ICD therapy and appropriate ICD shock were $17 \%$ (109 patients) and $10 \%$ (68 patients), respectively. Ischaemic aetiology and decreased LVEF $(<25 \%)$ remained independent risk factors for appropriate ICD shock.

A history of atrial tachyarrhythmia was a predictor for inappropriate ICD shock (HR 4.8, 95 \% CI: 2.4-9.7, $p<0.001)$. 
Table 1 Overview of all de novo implantations of implantable cardioverter-defibrillators performed at the UMCU over the years 2006-2011

\begin{tabular}{|c|c|c|c|c|c|}
\hline Characteristic & $\begin{array}{l}\text { Total population } \\
(n=1075) \\
\text { Number }(\%)\end{array}$ & $\begin{array}{l}\text { Ischaemic } \\
(n=626) \\
\text { Number }(\%)\end{array}$ & $\begin{array}{l}\text { Non-ischaemic } \\
(n=449) \\
\text { Number }(\%)\end{array}$ & $\begin{array}{l}\text { Secondary } \\
(n=421) \\
\text { Number }(\%)\end{array}$ & $\begin{array}{l}\text { Primary } \\
(n=654) \\
\text { Number }(\%)\end{array}$ \\
\hline \multicolumn{6}{|l|}{ Implantations } \\
\hline $2006 / 2007 / 2008 /$ & $134 / 144 / 148 /$ & $80 / 97 / 93 /$ & $54 / 47 / 55 /$ & $57 / 67 / 67 /$ & $77 / 77 / 81 /$ \\
\hline 2009/2010/2011 & $213 / 207 / 229$ & $112 / 113 / 131$ & $101 / 94 / 98$ & $86 / 60 / 71$ & $127 / 147 / 158$ \\
\hline $1 / 2 / 3$ leads & $596 / 165 / 314$ & $379 / 97 / 150$ & $217 / 68 / 164$ & $281 / 82 / 58$ & $315 / 83 / 256$ \\
\hline Ischaemic/non-ischaemic aetiology & $626(58) / 449(42)$ & - & - & $271(64) / 150(36)$ & $355(54) / 299(46) *$ \\
\hline Primary/secondary indication & $654(61) / 421(39)$ & $355(57) / 271(43)$ & $299(47) / 150(33)^{*}$ & - & - \\
\hline \multicolumn{6}{|l|}{ Demographics } \\
\hline Age(years, mean, SD) & $61 \pm 13$ & $66 \pm 10$ & $55 \pm 15^{*}$ & $62 \pm 14$ & $61 \pm 13$ \\
\hline Sex (male/female) & $796(74) / 279(26)$ & $526(84) / 100(16)$ & $270(60) / 179(40)^{*}$ & $337(80) / 84(20)$ & $459(70) / 195(30)^{*}$ \\
\hline \multicolumn{6}{|l|}{ Clinical parameters } \\
\hline LVEF (\%; mean, SD) & $30 \pm 13$ & $28 \pm 10$ & $33 \pm 17^{*}$ & $36 \pm 15$ & $27 \pm 11 *$ \\
\hline NYHA class (I/II/III/IV) & $75 / 194 / 267 / 14$ & $43 / 141 / 140 / 4$ & $32 / 53 / 127 / 10$ & $29 / 45 / 39 / 4$ & $46 / 149 / 228 / 10$ \\
\hline QRS duration (ms, mean, SD) & $127 \pm 33$ & $125 \pm 29$ & $130 \pm 37 *$ & $120 \pm 30$ & $132 \pm 34^{*}$ \\
\hline Diabetes mellitus II & 204(19) & $156(25)$ & $48(11)^{*}$ & $66(16)$ & $138(21)^{*}$ \\
\hline GFR (mean, SD) & $67 \pm 22$ & $65 \pm 20$ & $72 \pm 23 *$ & $71 \pm 22$ & $65 \pm 21$ \\
\hline History of atrial tachyarrhythmia & $300(28)$ & $174(28)$ & $126(28)$ & $125(30)$ & $175(27)$ \\
\hline Loss to follow-up & $116(11)$ & 67 & 49 & 63 & $53^{*}$ \\
\hline \multicolumn{6}{|l|}{ Medication at baseline } \\
\hline Antiarrhythmic agent & $842(78)$ & 520 & $322 *$ & 317 & $525^{*}$ \\
\hline Class II ${ }^{\text {a }}$ & $705(66)$ & 436 & $269^{*}$ & 452 & 253 \\
\hline Class III ${ }^{\mathrm{a}}$ & $102(9)$ & 88 & 53 & 59 & 82 \\
\hline
\end{tabular}

Mean follow-up period $31 \pm 17$ months

$S D$ standard deviation, $L V E F$ left ventricular ejection fraction, NYHA New York Heart Association, OHCA out-of-hospital cardiac arrest, GFR glomerular filtration rate (MDRD)

$* p<0.05$

${ }^{a}$ Alone or in combination with another antiarrhythmic drug

\section{Mortality}

Of the 155 patients who died, 50 patients (5\%) died within the first year after implantation. Over the follow-up period of $31 \pm 17$ months, 104 patients $(10 \%)$ died without receiving prior appropriate ICD therapy.

The yearly mortality rate was $5.6 \%$. The most important risk factors for mortality were: decreased GFR $(\leq 60)(\mathrm{HR} 2.3$, $95 \%$ CI: $1.6-3.2 ; p<0.001)$, a decreased LVEF ( $\leq 25 \%$ ) (HR $2.1,95 \%$ CI: $1.5-3.0 ; p<0.001$ ) and the male gender (HR 1.9, $95 \%$ CI: $1.3-3.0 ; p=0.004)$. In patients with a primary indication, a prolonged QRS duration ( $>130 \mathrm{~ms})$ was an additional risk factor for mortality. In patients with ICM, diabetes mellitus type II, older age ( $>70$ years) and decreased LVEF $(<25 \%)$ were predictive of mortality, while the use of beta-blockers decreased the risk of mortality. In the nonischaemic population, decreased GFR $(<60)$ and decreased LVEF $(<25 \%)$ were the most important risk factors. At baseline, decreased GFR $(<60)$, non-ischaemic aetiology and a history of AF were predictive of mortality within one year after primary prophylactic ICD implantation.

\section{Discussion}

Interpretation of and adherence to guidelines

\section{Baseline characteristics}

The demographics of our patient population are comparable with the characteristics of patients enrolled in the large randomised controlled trials $[3,6,8]$. Since the indication for ICD therapy is determined on the basis of these large trials, this seems a logical consequence, but it is worth mentioning that patients participating in randomised controlled trials may not be representative of patients typically seen in clinical practice. Only the incidence of a history of atrial tachyarrhythmias was substantially higher in our 

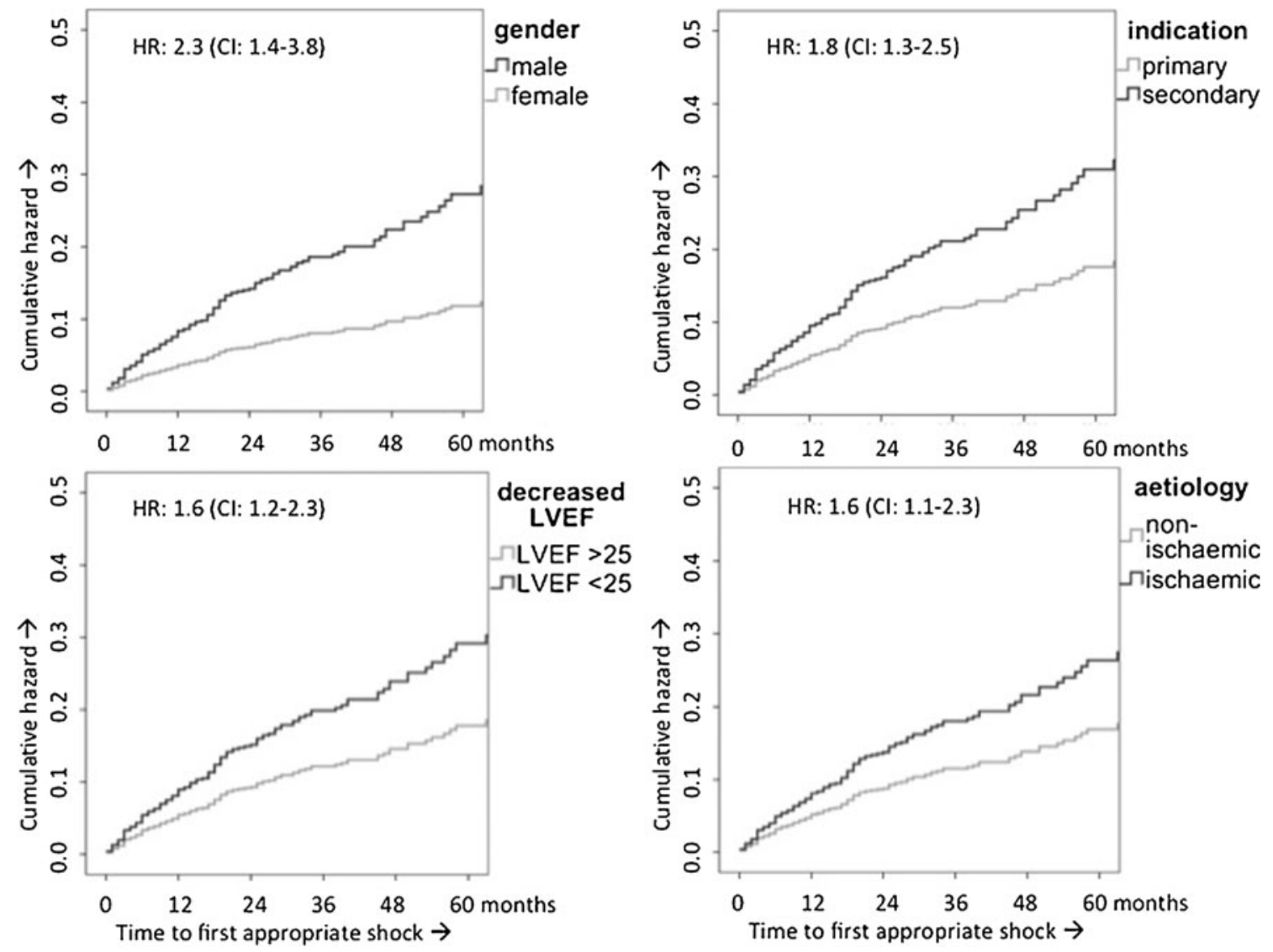

Fig. 1 Cumulative hazard for appropriate shock over time divided by different subpopulations

population since this was an exclusion criteria in most of these trials.

When we divide our population on the basis of aetiology, we observed that patients with ICM were older, predominantly male and had more comorbidities. The differences seen between LVEF in the ischaemic and non-ischaemic group can be explained by the fact that in a substantial number of the patients with NICM the indication for ICD implantation was not solely based on LVEF. A large proportion of patients with NICM have an underlying heart disease other than dilated cardiomyopathy, for whom risk factors such as unexplained syncope or hereditary taint (e.g. ARVC, LQTS) are more decisive when considering ICD implantation. Furthermore, NICM is more frequent in younger individuals and is associated somewhat more often with the female gender [21]. In our study we observed a more or less equal distribution between the two genders and a lower age at implant in the non-ischaemic population. The patients with a hereditary heart disease, such as ARVC, often receive an ICD early in life, which can attribute to the latter.

\section{Increase in the number of implantations}

As expected we observed an increase (171\%) in the number of implantations over the years 2006-2011. As mentioned before, this increase in (primary indications for) implantations can be explained by the implementation of large randomised controlled trials following the latest guidelines of 2008, which led to a broadening of indications [9].

Although we see a steady increase in implantations, we would have expected an even larger increase. On one hand, we could explain the limited increase by lack of adherence to the guidelines in clinical practice, but it can also represent a critical view on evidence and interpretation of these concomitant guidelines. Therefore, knowledge of current guidelines is crucial, not only to identify those eligible for ICD implantation but also those exempt from an indication [22, 23].

In the following paragraphs we will reflect on the incidences of inappropriate and appropriate ICD therapy and inappropriate and appropriate ICD shock and mortality. We should be careful about interpreting the absolute numbers since certain variables are time dependent. For example, a larger proportion of patients who received an ICD for primary prophylactic reasons received the ICD in the last couple of years of this study; therefore the follow-up of these patients will be relatively shorter than for the patients who received an ICD for secondary prophylaxis. With the multivariate Cox regression analysis, we corrected for this by taking the time to the (first) event into account. 


\section{Appropriate ICD therapy}

In the current study, 227 (21\%) patients of our total population received appropriate ICD therapy after a mean followup of $31 \pm 17$ months, which corresponds to an annual appropriate ICD therapy rate of $8.1 \%$. This number compares well with other registries $[24,25]$. We found an appropriate ICD shock rate of $14 \%$. A secondary indication, ischaemic aetiology, a decreased LVEF and a male gender were identified as independent risk factors for appropriate ICD shock. The annual appropriate ICD shock rate of $5.4 \%$ compares well with the findings of an earlier study by Van Welsenes et al., performed in Leiden, the Netherlands, over the years 1996-2008 [24]. In their population of 2134 patients with both primary and secondary indication, they found an annual appropriate ICD shock rate of $5.9 \%$. The appropriate ICD shock rate in our primary prophylactic population was lower with an annual appropriate ICD shock rate of $4 \%$. In the SCD-HeFT trial (patients with a primary indication and both ischaemic and non-ischaemic heart disease) an annual appropriate ICD shock rate of $5.1 \%$ was seen. Follow-up data from the DEFINITE trial (patients with a primary indication and non-ischaemic heart disease) showed an incidence of $7 \%$ of appropriate ICD shock after 29 months of follow-up (annual appropriate ICD shock rate $2.9 \%$ ). The incidence of appropriate ICD shock in our patients implanted for primary prophylaxis and non-ischaemic heart disease was $4 \%$ (annual appropriate ICD shock rate of $1.7 \%$ ). The somewhat higher 'appropriate' ICD shock rates in both the SCD-HeFT trial as well as the DEFINITE trial could be explained by the ICD settings. In both trials ICDs were programmed with shock therapy only (no ATP) at a single zone of $>187$ beats $/ \mathrm{min}$ and $>180$ beats $/ \mathrm{min}$, respectively. After three years of follow-up of the MADIT II study, which consisted of patients with a primary indication and ischaemic heart disease, $20 \%$ of their patients experienced an appropriate ICD shock at least once [26]. In our primary prophylactic patients with ischaemic heart disease this rate was found to be $16 \%$ after approximately 2.5 years, which might be explained by the shorter follow-up period and by the unselected nature of our patient population in contrast to the large prospective trials such as MADIT II.

When we only select the patients who received an ICD for primary prophylaxis, a lower incidence of appropriate ICD therapy and ICD shocks was seen in the subpopulation with NICM compared with the ischaemic group. The DEFINITE and SCD-HeFT failed to reach statistical significance for the benefit of ICD implantation for primary prophylaxis in the subset of patients with NICM. Nevertheless, a trend toward reduced mortality was seen $[3,6]$. It should be noted that the DEFINITE trial demonstrated significant effectiveness of ICD therapy in NICM patients for preventing death from cardiac causes, but not all-cause death. The lack of statistical significance is probably due to low mortality in the NICM population that was also the reason for prematurely stopping the AMIOVIRT and CAT trial [27, 28]. Furthermore, a sub-analysis of the SCD-HeFT trial showed a relatively high mortality due to pump failure instead of arrhythmic death in patients with non-ischaemic cardiomyopathy and NYHA functional class III. This implies a relatively small absolute mortality benefit, and therefore a higher number needed to treat ( 25 versus 18 ICD implantations to prevent one death in two years for NICM patients and ICM patients respectively) [29]. Furthermore, if these patients receive appropriate ICD therapy, the question is: 'how many life years can be gained due to appropriate ICD therapy?' In patients with end-stage heart failure recurrent VTs can be a sign of progression of the impaired LV function. Although the ICD can successfully treat ventricular tachyarrhythmias, it cannot prevent death from pump failure. It is also important to realise that ICD shock, and certainly ICD therapy, cannot be replaced $1: 1$ by a prevented sudden cardiac death. Multiple trials have shown that the incidence of ICD shocks is substantially higher than the incidence of sudden cardiac death.

As described in our results the male gender was one of the independent risk factors for appropriate ICD therapy and ICD shock in our population. When we divide the population based on aetiology or indication, patients with ischaemic heart disease and patients with an ICD implanted for secondary reasons received the most appropriate ICD shocks. The male gender was more common in those subpopulations. However, when we corrected for these variables, males still had a higher probability to receive an appropriate ICD shock (HR 2.3, $95 \%$ CI: 1.4-3.8; $p=0.001$ ). The registry of Wilson et al. and the follow-up study of MADIT II also found a higher number of men in their appropriate ICD shock population $[26,30]$. Moreover, the long-term follow-up study of the MADIT II cohort showed a smaller benefit of ICD implantation in women [31]. Differences in repolarisation and in arrhythmogenic substrate are suggested to cause this difference in benefit of ICD implantation [32-34]. Risk factors for appropriate ICD therapy could therefore be different in the female population. Since some studies also reported a higher complication rate in females, it could be of importance to further elucidate whether this should have consequences for patient selection and risk stratification [35].

When we exclude patients who received an ICD for secondary prophylaxis from the analysis, aetiology and decreased LVEF remained independent risk factors for appropriate ICD shock. Only gender was no longer an independent risk factor though the incidence of appropriate ICD shock in females was significantly lower than in males in patients with an ICD implanted for primary prophylaxis. The low number of females and subsequent low number of events in this subpopulation probably caused the analysis to be statistically 
underpowered. This supposition was supported by the fact that when we analysed the risk factors for appropriate ICD therapy (higher number of events), gender was an independent risk factor for appropriate ICD therapy.

\section{Inappropriate ICD therapy}

Inappropriate ICD therapy, and inappropriate ICD shock in particular, leads to an impaired quality of life and psychological and psychiatric effects $[36,37]$. As a matter of course inappropriate ICD therapy must be prevented to the extent possible. In our population we found an inappropriate ICD shock rate of $3 \%$ after $31 \pm 17$ months of follow-up. This number is much lower compared with other registries or the landmark trials. In the study by Van Welsenes et al., $14 \%$ of their patients experienced an inappropriate ICD shock $[24,38]$. The follow-up studies of SCD-HeFT, DEFINITE and MADIT II found inappropriate ICD shock rates of $10 \%, 10 \%$ and $11.5 \%$ after 45,29 and 20 months of follow-up, respectively $[3,39,40]$. In the MADIT II study and the registry of Van Welsenes et al. prior atrial fibrillation was predictive of inappropriate ICD shock [24, 38, 40]. Our study confirms prior atrial tachyarrhythmia to be a risk factor for inappropriate ICD shock (HR 4.8, 95 \% CI: $2.4-$ 9.7, $p<0.001)$ and also patients with NICM received a higher number of inappropriate ICD shocks (HR 2.1, $95 \%$ CI:1.0-4.1, $p<0.038)$. In other studies a higher incidence of inappropriate ICD shocks was seen in younger patients and patients with renal dysfunction [40, 41]. Regarding the low incidence of inappropriate ICD shock in our population, it is important to emphasise that the number of inappropriate ICD shocks is greatly dependent on device settings. At the UMCU our philosophy is to program the device only for the treatment of fast VT or VF (life-threatening tachyarrhythmias); slow VTs (haemodynamically stable VTs) were treated by VT ablation or pharmacologically. Furthermore, SVT/VT discriminators are enabled in the VT zones of all patients. The more than double inappropriate ICD shock rate in the SCD-HeFT trial, for example, can be related to the fact that all ICDs were programmed with shock therapy at a single zone of $>187$ beats/min without discrimination between VT and SVT [3].

\section{Risk factors for mortality}

One prerequisite of ICD implantation is a life expectancy of more than 1 year. Furthermore an ICD will only be feasible if appropriate ICD therapy is delivered before the patient dies. Estimating risk at death is therefore useful and often already an important consideration in clinical practice. In our population, 50 patients died in the first year after implantation, and therefore, in retrospect, were not eligible for ICD treatment. Furthermore, $10 \%$ of the patients died without receiving appropriate ICD therapy. Different factors were associated with death in the different subpopulations. The risk factors we found were comparable with other registries [42, 43]. In our study impaired renal function was the most important risk factor for mortality.

\section{Study limitations}

In this analysis there was no control population available. Hence, we could not compare the adherence to guidelines in patients with and without ICD implantation. Furthermore, we performed a retrospective study, which entails some disadvantages. All the analysed data were retrieved from electronic medical records. Some information was not recorded in the medical records of all patients, such as NYHA class and LVEF. We noticed that in the 58 cases in which the quantification of LVEF in percentages was lacking, the LVEF was predominantly described as normal or good. This could probably cause bias. To calculate the average LVEF we used 1017 cases in which LVEF was quantified as a percentage. For the multivariate Cox regression analysis, in which we used LVEF $<25 \%$ as a variable, we used 41 more cases (1058). In these 41 cases we could be certain, based on the echocardiogram quantification and medical records, that these patients had to have an LVEF $>25 \%$. In this way the probable bias that can occur based on the abovementioned is avoided.

We collected recent data, which led to a relatively short follow-up period. The loss of follow-up was mainly caused by the fact that some patients, mostly elderly, return to their own cardiologist in the referral hospital for routine check-up.

\section{ICD implantation: where do we go from here?}

Decreased LVEF has shown to have an inverse relationship with cardiac mortality and approximately $50 \%$ of the cardiac deaths are a result of sudden death. Since no other reliable methods are available for identifying in advance those patients who are more likely to die from sudden death, LVEF is considered a valuable criterion for ICD implantation $[1,3,44]$.

Due to trial designs, left ventricular dysfunction has developed into a key determinant for the selection of candidates for prophylactic ICD implantation, even though a causal relation between LVEF and the pathophysiology of ventricular arrhythmias is not clearly demonstrated. Furthermore, prior research has shown that the largest proportion of non-survivors of out-of-hospital cardiac arrest had an LVEF of $30 \%$ or higher prior to the event, and would not have been eligible for prophylactic ICD implantation [45, 46]. The relative risk for ventricular tachyarrhythmias in people with a normal LVEF is much lower and the number to treat very high. In our population the mean LVEF of patients with 
a secondary indication was $36 \pm 15 \%$, comparable with the three large secondary prevention trials which presented a mean LVEF after out-of-hospital cardiac arrest of 32\%-45\% $[2,5,7]$. Note that the patients with an ICD implanted for secondary prophylaxis had a significantly higher number of appropriate ICD shocks. On the other hand, impaired LVEF $(<25 \%)$ was an independent risk factor for appropriate ICD therapy and ICD shock in the overall population and also in the subgroup of patients implanted for primary prophylaxis.

As we can see in our population, patients with NICM, patients with an ICD for primary prophylactic reasons, and women experienced the lowest number of appropriate ICD shocks, and therefore have the least benefit from ICD implantation. At the moment, ICD implantation in these groups is mainly based on the LVEF. Improvement of selection of eligible patients by electrophysiological parameters has been studied, but these studies often did not find a hazard ratio greater than two. Furthermore, electrophysiological tests are not feasible in all candidates for prophylactic ICD implantation [47, 48]. The Alternans Before Cardiac Defibrillator trial (ABCD trial [49]) was the first to suggest to combine different electrophysiological parameters to increase the predictive power to guide ICD therapy. The large prospective European multicentre study, EUTrigTreat, embraces this idea. The study enrols patients with standard indications for ICD implantation in a population consisting of both ischaemic and non-ischaemic heart disease. It is an observational trial with the aim to accurately stratify ICD patients, who are at risk for ICD shock and mortality using traditional risk markers as well as genetic markers. It compares the predictive power and temporal changes of various invasive and non-invasive electrophysiological tests such as programmed ventricular stimulation (PVS), T-wave alternans (TWA), beat-to-beat variability of repolarisation quantified as short-term variability (STV), heart rate variability (HRV) and heart rate turbulence (HRT) to determine which patients will be at risk for life-threatening arrhythmias and will benefit from ICD implantation [50, 51].

\section{Conclusion}

As in all Western European countries we experienced an increase in the number of ICD implantations mainly based on the increase in primary prophylactic implantations. The rate of appropriate ICD shocks in our unselected population corresponds to the large clinical trials, which confirms the benefit of ICD implantation when following current guidelines. Nonetheless a significantly lower rate of appropriate ICD shocks were observed in patients implanted for primary prevention, patients with non-ischaemic cardiomyopathy and women. This implies a lower ICD benefit or at least a higher number needed to treat in these subpopulations. It would be lucrative to improve the risk stratification and patient selection for ICD implantation to accomplish optimal clinical benefit and cost-effectiveness. The prospective European multicentre study, EUTrigTreat, might contribute to this by a more patient-tailored risk stratification based on electrophysiological parameters. In our population few patients (only $3 \%$ ) received an inappropriate ICD shock; we attribute this to adequate ICD programming and alternative treatment of slow VTs.

Funding The research leading to the results received funding from the European Community's Seventh Framework Program FP7/2007-2013 under grant agreement No. HEALTH-F2-2009-241526, EUTrigTreat.

\section{Conflict of interests None declared.}

Open Access This article is distributed under the terms of the Creative Commons Attribution License which permits any use, distribution, and reproduction in any medium, provided the original author(s) and the source are credited.

\section{References}

1. Moss AJ, Hall WJ, Cannom DS, et al. Improved survival with an implanted defibrillator in patients with coronary disease at high risk for ventricular arrhythmia. Multicenter automatic defibrillator implantation trial investigators. N Engl J Med. 1996;335(26):1933-40.

2. Antiarrhythmics versus Implantable Defibrillators (AVID) investigators. A comparison of antiarrhythmic-drug therapy with implantable defibrillators in patients resuscitated from near-fatal ventricular arrhythmias. N Engl J Med. 1997 Nov 27;337(22):1576-83

3. Bardy GH, Lee KL, Mark DB, et al. Amiodarone or an implantable cardioverter-defibrillator for congestive heart failure. $\mathrm{N}$ Engl $\mathrm{J}$ Med. 2005;352(3):225-37.

4. Buxton AE, Lee KL, Fisher JD, et al. A randomized study of the prevention of sudden death in patients with coronary artery disease. Multicenter unsustained tachycardia trial investigators. N Engl J Med. 1999;341(25):1882-90.

5. Connolly SJ, Gent M, Roberts RS, et al. Canadian Implantable Defibrillator Study (CIDS): a randomized trial of the implantable cardioverter defibrillator against amiodarone. Circulation. 2000;101(11):1297-302.

6. Kadish A, Dyer A, Daubert JP, et al. Prophylactic defibrillator implantation in patients with nonischemic dilated cardiomyopathy. N Engl J Med. 2004;350(21):2151-8.

7. Kuck KH, Cappato R, Siebels J, et al. Randomized comparison of antiarrhythmic drug therapy with implantable defibrillators in patients resuscitated from cardiac arrest: the Cardiac Arrest Study Hamburg (CASH). Circulation. 2000;102(7):748-54.

8. Moss AJ, Zareba W, Hall WJ, et al. Prophylactic implantation of a defibrillator in patients with myocardial infarction and reduced ejection fraction. N Engl J Med. 2002;346(12):877-83.

9. Epstein AE, DiMarco JP, Ellenbogen KA, et al. ACC/AHA/HRS 2008 guidelines for device-based therapy of cardiac rhythm Abnormalities: a report of the American college of cardiology/ American heart association task force on practice guidelines (writing committee to revise the ACC/AHA/NASPE 2002 guideline update for implantation of cardiac pacemakers and antiarrhythmia devices) 
developed in collaboration with the American association for thoracic surgery and society of thoracic surgeons. J Am Coll Cardiol. 2008;51(21):e1-e62.

10. van Erven L, van Dessel PFHM, Simmers TA, et al. Guidelines ICD implantation 2005 - an update. The Netherlands Society of Cardiology (Nederlandse Vereniging voor Cardiologie), version April 2006.

11. Zipes DP, Camm AJ, Borggrefe M, et al. ACC/AHA/ESC 2006 guidelines for management of patients with ventricular arrhythmias and the prevention of sudden cardiac death: a report of the American college of cardiology/american heart association task force and the European society of cardiology committee for practice guidelines (writing committee to develop guidelines for management of patients with ventricular arrhythmias and the prevention of sudden cardiac death) developed in collaboration with the European heart rhythm association and the heart rhythm society. Europace. 2006;8(9):746837.

12. Mark DB, Nelson CL, Anstrom KJ, et al. Cost-effectiveness of defibrillator therapy or amiodarone in chronic stable heart failure: results from the Sudden Cardiac Death in Heart Failure Trial (SCD-HeFT). Circulation. 2006;114(2):135-42.

13. Zwanziger J, Hall WJ, Dick AW, et al. The cost effectiveness of implantable cardioverter-defibrillators: results from the Multicenter Automatic Defibrillator Implantation Trial (MADIT)II. J Am Coll Cardiol. 2006;47(11):2310-8.

14. Cowie MR, Marshall D, Drummond M, et al. Lifetime costeffectiveness of prophylactic implantation of a cardioverter defibrillator in patients with reduced left ventricular systolic function: results of Markov modelling in a European population. Europace. 2009;11(6):716-26

15. Smith T, Jordaens L, Theuns DA, et al. The cost-effectiveness of primary prophylactic implantable defibrillator therapy in patients with ischaemic or non-ischaemic heart disease: a European analysis. Eur Heart J. 2013;34(3):211-9.

16. Bracke FA, Dekker LR, van der Voort PH, et al. Primary prevention with the ICD in clinical practice: not as straightforward as the guidelines suggest? Neth Heart J. 2009;17(3):107-10.

17. van Welsenes GH, Borleffs CJ, van Rees JB, et al. Improvements in 25 Years of implantable cardioverter defibrillator therapy. Neth Heart J. 2011;19(1):24-30.

18. Zipes DP, Camm AJ, Borggrefe M, et al. ACC/AHA/ESC 2006 guidelines for management of patients with ventricular arrhythmias and the prevention of sudden cardiac death: a report of the American college of cardiology/American heart association task force and the European society of cardiology committee for practice Guidelines (writing committee to develop guidelines for management of patients with ventricular arrhythmias and the prevention of sudden cardiac death). J Am Coll Cardiol. 2006;48(5):e247-346.

19. Levey AS, Bosch JP, Lewis JB, et al. A more accurate method to estimate glomerular filtration rate from serum creatinine: a new prediction equation. Modification of diet in renal disease study group. Ann Intern Med. 1999;130(6):461-70.

20. Vaughan Williams EM. Classification of antidysrhythmic drugs. Pharmacol Ther B. 1975;1(1):115-38.

21. Follath F. Nonischemic heart failure: epidemiology, pathophysiology, and progression of disease. J Cardiovasc Pharmacol. 1999;33 Suppl 3:S31-5.

22. Borleffs CJ, Wilde AA, Cramer MJ, et al. Clinical implementation of guidelines for cardioverter defibrillator implantation: lost in translation? Neth Heart J. 2007;15(4):129-32.

23. Al-Khatib SM, Sanders GD, Carlson M, et al. Preventing tomorrow's sudden cardiac death today: dissemination of effective therapies for sudden cardiac death prevention. Am Hear J. 2008;156(4):613-22.

24. van Welsenes GH, van Rees JB, Borleffs CJ, et al. Long-term follow-up of primary and secondary prevention implantable cardioverter defibrillator patients. Europace. 2011;13(3):389-94.
25. Smith T, van Dessel PF, Theuns DA, et al. Health care utilisation after defibrillator implantation for primary prevention according to the guidelines in 2 Dutch academic medical centres. Neth Heart J. 2011;19(10):405-11.

26. Moss AJ, Greenberg H, Case RB, et al. Long-term clinical course of patients after termination of ventricular tachyarrhythmia by an implanted defibrillator. Circulation. 2004;110(25):3760-5.

27. Bansch D, Antz M, Boczor S, et al. Primary prevention of sudden cardiac death in idiopathic dilated cardiomyopathy: the Cardiomyopathy Trial (CAT). Circulation. 2002;105(12):1453-8.

28. Strickberger SA, Hummel JD, Bartlett TG, et al. Amiodarone versus implantable cardioverter-defibrillator:randomized trial in patients with nonischemic dilated cardiomyopathy and asymptomatic nonsustained ventricular tachycardia-AMIOVIRT. J Am Coll Cardiol. 2003;41(10):1707-12.

29. Desai AS, Fang JC, Maisel WH, et al. Implantable defibrillators for the prevention of mortality in patients with nonischemic cardiomyopathy: a meta-analysis of randomized controlled trials. Jama. 2004;292(23):2874-9.

30. Wilson D, Shi B, Harding S, et al. Implantable cardioverterdefibrillators: a long-term view. Intern Med J. 2012;42(5):554-61 [Research Support, Non-U.S. Gov't].

31. Goldenberg I, Gillespie J, Moss AJ, et al. Long-term benefit of primary prevention with an implantable cardioverter-defibrillator: an extended 8-year follow-up study of the multicenter automatic defibrillator implantation trial II. Circulation. 2010;122(13):1265-71.

32. Santangeli P, Pelargonio G, Dello Russo A, et al. Gender differences in clinical outcome and primary prevention defibrillator benefit in patients with severe left ventricular dysfunction: a systematic review and meta-analysis. Hear Rhythm. 2010;7(7):876-82.

33. Haigney MC, Zareba W, Nasir JM, et al. Gender differences and risk of ventricular tachycardia or ventricular fibrillation. Hear Rhythm. 2009;6(2):180-6.

34. Ghanbari H, Dalloul G, Hasan R, et al. Effectiveness of implantable cardioverter-defibrillators for the primary prevention of sudden cardiac death in women with advanced heart failure: a meta-analysis of randomized controlled trials. Arch Intern Med. 2009;169(16):1500-6.

35. MacFadden DR, Crystal E, Krahn AD, et al. Sex differences in implantable cardioverter-defibrillator outcomes: findings from a prospective defibrillator database. Ann Intern Med. 2012;156(3):195203.

36. Heller SS, Ormont MA, Lidagoster L, et al. Psychosocial outcome after ICD implantation: a current perspective. Pacing Clin Electrophysiol. 1998;21(6):1207-15.

37. Schron EB, Exner DV, Yao Q, et al. Quality of life in the antiarrhythmics versus implantable defibrillators trial: impact of therapy and influence of adverse symptoms and defibrillator shocks. Circulation. 2002;105(5):589-94.

38. van Rees JB, Borleffs CJ, de Bie MK, et al. Inappropriate implantable cardioverter-defibrillator shocks: incidence, predictors, and impact on mortality. J Am Coll Cardiol. 2011;57(5):556-62.

39. Ellenbogen KA, Levine JH, Berger RD, et al. Are implantable cardioverter defibrillator shocks a surrogate for sudden cardiac death in patients with nonischemic cardiomyopathy? Circulation. 2006;113(6):776-82.

40. Daubert JP, Zareba W, Cannom DS, et al. Inappropriate implantable cardioverter-defibrillator shocks in MADIT II: frequency, mechanisms, predictors, and survival impact. J Am Coll Cardiol. 2008;51(14):1357-65.

41. Alter P, Waldhans S, Plachta E, et al. Complications of implantable cardioverter defibrillator therapy in 440 consecutive patients. Pacing Clin Electrophysiol. 2005;28(9):926-32.

42. Borleffs CJ, van Welsenes GH, van Bommel RJ, et al. Mortality risk score in primary prevention implantable cardioverter defibrillator recipients with non-ischaemic or ischaemic heart disease. Eur Heart J. 2010;31(6):712-8. 
43. van Rees JB, Borleffs CJ, van Welsenes GH, et al. Clinical prediction model for death prior to appropriate therapy in primary prevention implantable cardioverter defibrillator patients with ischaemic heart disease: the FADES risk score. Heart. 2012;98(11):872-7.

44. Multicenter Postinfarction Research Group. Risk stratification and survival after myocardial infarction. N Engl J Med. 1983 Aug $11 ; 309(6): 331-6$.

45. Wilde AA, Simmers TA. Primary prevention with ICDs, are we on the right track? Neth Heart J. 2009;17(3):92-4.

46. de Vreede-Swagemakers JJ, Gorgels AP, Dubois-Arbouw WI, et al. Out-of-hospital cardiac arrest in the 1990's: a population-based study in the Maastricht area on incidence, characteristics and survival. J Am Coll Cardiol. 1997;30(6):1500-5.

47. Jackson CE, Myles RC, Tsorlalis IK, et al. Profile of microvolt T-wave alternans testing in 1003 patients hospitalized with heart failure. Eur J Heart Fail. 2012;14(4):377-86 [Research Support, Non-U.S. Gov't].
48. Kraaier K, McCracken T, van der Palen J, et al. Is T-wave alternans testing feasible in candidates for prophylactic implantable defibrillators? Neth Heart J. 2011;19(1):6-9.

49. Amit G, Rosenbaum DS, Super DM, et al. Microvolt T-wave alternans and electrophysiologic testing predict distinct arrhythmia substrates: implications for identifying patients at risk for sudden cardiac death. Hear Rhythm. 2010;7(6):7638.

50. Seegers J, Vos MA, Flevari P, et al. Rationale, objectives, and design of the EUTrigTreat clinical study: a prospective observational study for arrhythmia risk stratification and assessment of interrelationships among repolarization markers and genotype. Europace. 2012;14(3):416-22.

51. Varkevisser R, Wijers SC, van der Heyden MA, et al. Beat-to-beat variability of repolarization as a new biomarker for proarrhythmia in vivo. Hear Rhythm. 2012;9(10):1718-26.

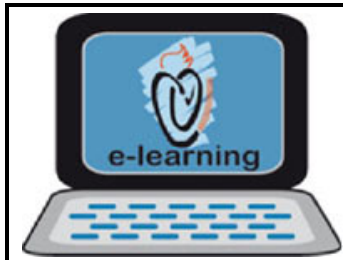

\section{CVOI E-learning formula!}

This is the CVOI e-learning article. The author has prepared 10 questions which are available through the website of the Cardiovascular Educational Institute (CVOI). Please follow the instructions below.

After finishing the questions you will be asked to fill in your name, hospital and e-mail address; then press the button 'verzenden'.

When 6 out of the 10 questions are answered correctly, you acquire 1 accreditation point granted by the Quality Committee of the Netherlands Society of Cardiology (NVVC). The acquired point will be credited to your personal file in the GAIA system. You will also receive an e-mail with all the correct answers.

Over a period of one year 10 e-learning articles will appear in 10 subsequent $\mathrm{NHJ}$ editions. In each edition the e-learning article will be recognisable by a special icon. On an annual basis you can collect 10 accreditation points. The accreditation points are credited in the GAIA system by the CVOI.

If you need additional information, please contact the CVOI by e-mail: cvoi@cvoi.org or by phone: 030-2345001.

E.E. van der Wall

Chief editor NHJ
K.B. Schick

Coordinator CVOI 